\title{
Visual and auditory evoked responses in patients with Parkinson's disease
}

\author{
M J G A W E, P DAS, S VINCENT, AND F CLIFFORD ROSE \\ From the Departments of Neurology, and Medical Physics, Charing Cross Hospital, London
}

SUMMARY Visual evoked responses were obtained in 47 Parkinsonian patients and 26 agematched controls. The stimulation to binocular and uniocular latency of the major positive peak was longer in the Parkinsonian patients and its amplitude was smaller than in the control subjects. There was a large interocular difference suggesting that at least part of the delay occurs at prechiasmatic level. The latency and amplitude varied markedly following ingestion of levodopa as Sinemet. ${ }^{(k)}$ Auditory evoked potentials were obtained in 16 Parkinsonian patients and 11 age-matched controls. The latency of the NV wave was prolonged in the former although the amplitude was the same, suggesting that the abnormality may be a widespread one.

The techniques of evoked responses have been used in the detection of subclinical lesions in optic nerve, spinal cord and brain stem in the diagnosis of multiple sclerosis. ${ }^{12}$ It has become clear that factors other than demyelination can affect the shape and latency of the waveforms. These techniques can be used to probe function in the nervous system rather than be confined to identifying structural abnormalities. Celesia and Daly ${ }^{3}$ found that the latency of the first negative and the major pcsitive wave of the visual evoked potential (VEP) increased with age. This raises the possibility that the study of VEPs in disorders which were perhaps characterised by accelerated senescence such as Parkinson's disease could be of value. The finding by Schafer and $\mathrm{McKean}^{4}$ that phenylketonuric children have abnormally delayed VEP latencies which become normal upon treatment with a phenylalanine free diet or the addition to the diet of indole and catecholamine precursors suggested that catecholamine played a part in the formation of the evoked potential. Bodis Wollner and $\mathrm{Yahr}^{5}$ demonstrated an abnormally delayed latency of the major positive wave in patients with Parkinson's disease. This delay was shortened in some patients following levodopa therapy indicating that at least some part of the abnormality causing it was amenable to pharmacological manipulation. Their original experiments were performed using a grat-

Address for reprint requests: Dr MJ Gawel, Department of Neurology, Charing Cross Hospital, London W6 8RF.

Accepted 20 November 1980 ing as the stimulus but in subsequent experiments they were able to reproduce their findings using a reversing checkerboard. They also correlated severity of disease with prolongation of latency. In their paper they only examined monocular latency of the major positive wave on stimulation of each eye, but since binocular stimulation activates different pathways and measurement of amplitude of response has relevance in VEP studies it seemed to us that these factors needed study in patients with Parkinson's disease. Bodis Wollner and Yahr's ${ }^{5}$ paper also showed that there was an interocular difference in the latency of the major positive wave in Parkinsonian patients and that in some patients treatment altered latency in one eye only. This suggested to them that the delay was prechiasmatic and possibly retinal where there are dopaminergic cells in the interplexiform layer which appear to have $\mathrm{D} 1$ receptor response characteristics. ${ }^{6}$ It has been suggested that the influence of amines on evoked potentials is exerted at cortical levels. ${ }^{4-9}$ Further evidence for a more generalised effect would be obtained if a delay could be demonstrated upon stimulation of another modality for instance auditory. We studied both visual and auditory evoked responses in patients with Parkinson's disease comparing them with normal age matched controls.

\section{Materials and methods}

The patients (table 1) were attending the Charing Cross Parkinson's disease clinic of the Department of 
Neurology. Controls were either the spouses of the patients or other individuals working in the hospital of a suitable age. All subjects had visual acuity equivalent to or better than $\mathrm{J} 4$ (corrected), normal visual fields and were free from any ocular disorder. None had a past history of cardiovascular disease or multiple sclerosis. There were 47 patients (mean age $67 \mathrm{yr}$, age range 51-82, 30 males and 17 females), and 26 controls (mean age $63 \mathrm{yr}$, age range 40-90, 11 males and 15 females) in the VEP study and 21 patients and 12 controls in the auditory evoked potential (AER) study. Thirteen of the patients in the AER study had also had VEPs. All but 11 of the patients who had VEPs performed were on levodopa therapy and in these the studies were performed as near as possible to two hours after the previous dose. Eleven patients were newly diagnosed and had VEP performed before starting treatment.

VEPs were obtained using a reversing checkerboard stimulus (Digitimer Mark II) giving a luminance of $6.86 \mathrm{~cd} \mathrm{~m}^{-2}$ for the light squares and $1.46 \mathrm{~cd} \mathrm{~m}^{-2}$ for the dark squares in the centre of the field. Each square was $1 \mathrm{~cm}$ square and the patient was positioned 1 metre away. Pattern reversal occurred every $500 \mathrm{~ms}$ and the responses were averaged for 96 sweeps in each monocular and binocular stimulation. The recording electrode was in the midline and $5 \mathrm{~cm}$ above the inion with the reference $20 \mathrm{~cm}$ anterior to it. The potentials obtained were displayed on an oscilloscope screen and the P2 latency was measured automatically using a movable cursor. Amplitudes of the $\mathbf{P} 2$ waves were measured from images of the potentials on light sensitive paper with reference to a calibration signal of $10 \mu \mathrm{v}$. The measurements taken were as follows: Latency P2 was the latency (in ms) from the onset of reversal to the major positive peak (P2). Amplitude $A_{1}$, was the amplitude from the first negative wave $\mathrm{N}$, to the major positive peak P2. Amplitude A2 was from P2 to the following negative wave $\mathrm{N} 2$.

Auditory evoked responses were obtained using a click stimulus $70 \mathrm{db}$ above threshold delivered at the rate of $10 / \mathrm{s}$ and averaged over 2000 repetitions. Recordings were from a mastoid electrode placement and the following measurements taken: the latency of the fifth negative wave NV was from the onset of the stimulus to the first negative peak. Amplitude of NV was from NV to the following positive peak. The latency and amplitude of the first negative peak NI was also measured. Results were analysed using Student's $t$ test on individual measurements and a multivariate analysis (RAO) on the amplitudes and latencies of the VEP.

\section{Results}

(1) VISUAL EVOKED POTENTIALS

These are summarised in tables 1,2 and 3. Amplitude and latency were different in patients with Parkinson's disease when taken as a group. Com-
Table 1 VEP in Parkinson's disease-comparison within the groups of binocular, left and right eye latencies

\begin{tabular}{|c|c|c|c|}
\hline & & Patients & Controls \\
\hline \multirow{2}{*}{$\begin{array}{l}\text { P2 Latency } \\
\text { (ms) }\end{array}$} & Binocular & $109 \cdot 21 \pm 13 \cdot 4$ & $100 \cdot 52 \pm 8 \cdot 7$ \\
\hline & $\begin{array}{l}\text { Left } \\
\text { Right }\end{array}$ & $\begin{array}{l}113 \cdot 5 \pm 16^{*} \\
112 \cdot 17 \pm 14 \dagger\end{array}$ & $\begin{array}{l}102 \cdot 64 \pm 8 \cdot 6_{+}^{+} \\
102 \cdot 93 \pm 8 \cdot 3_{+}^{+}\end{array}$ \\
\hline $\begin{array}{l}{ }^{*} \mathbf{P} 2 B \text { vs } \mathbf{P} 2 \mathbf{L} \\
\dagger \mathbf{P} 2 \mathbf{B} \text { vs } \mathbf{P} 2 \mathrm{R}\end{array}$ & $\begin{array}{l}=0.004 \\
=0.02\end{array}$ & $\begin{array}{l}\text { P2L } p=0.05 \\
\text { P2R p }=0.05\end{array}$ & \\
\hline
\end{tabular}

Table 2 VEP in Parkinson's disease

\begin{tabular}{llcccc}
\hline \multicolumn{5}{c}{ Patients $(47)$} & Controls $(21) p$ \\
\hline Binocular & P2 latency $(\mathrm{ms})$ & $109.2 \pm 13.4^{*}$ & $100.7 \pm 8.6$ & $<0.002$ \\
& A1 amplitude $(\mu \mathrm{v})$ & $7.6 \pm \pm 3.2$ & $14.3 \pm 5.7$ & $<0.001$ \\
& A2 amplitude $(\mu \mathrm{v})$ & $10.4 \pm 5.2$ & $17.4 \pm 8.8$ & $<0.01$ \\
Left eye & P2 latency $(\mathrm{ms})$ & $113.5 \pm 16$ & $102.6 \pm 8$ & $<0.001$ \\
& A1 amplitude $(\mu \mathrm{v})$ & $7.4 \pm 2.5$ & $13.8 \pm 6$ & $<0.002$ \\
& A2 amplitude $(\mu \mathrm{v})$ & $9.8 \pm 4.4$ & $18.4 \pm 12$ & $<0.05$ \\
Right eye & P2 latency $(\mathrm{ms})$ & $112.17 \pm 14$ & $102.9 \pm 8$ & $<0.001$ \\
& A1 amplitude $(\mu \mathrm{v})$ & $6.7 \pm 2.8$ & $12.8 \pm 3.8$ & $<0.001$ \\
& A2 amplitude $(\mu \mathrm{v})$ & $9.3 \pm 4.4$ & $17.5 \pm 10$ & $<0.008$ \\
\hline
\end{tabular}

$*$ SD.

Table 3 VEP in Parkinson's disease

\begin{tabular}{llll}
\hline Interocular differences & Patients & Controls & $p$ \\
\hline Difference of latencies (ms) & $6 \cdot 4 \pm 7 \cdot 4 *$ & $1 \cdot 8 \pm 2 \cdot 6$ & $<0 \cdot 001$ \\
Difference of amplitude A1 $(\mu \mathrm{v})$ & $1 \cdot 9 \pm 2 \cdot 9$ & $3 \cdot 9 \pm 5 \cdot 3$ & NS \\
Difference of amplitude A2 $(\mu \mathrm{v})$ & $2 \cdot 2 \pm 1 \cdot 8$ & $3 \cdot 9 \pm 4 \cdot 1$ & NS \\
\hline
\end{tabular}

\pm SD.

paring the treated and untreated patients no differences emerged so they have been treated as one group. The same relationship of faster conduction with binocular stimulation held in both control and Parkinsonian patients. This was found in migraine patients ${ }^{10}$ and warrants further study. In the Parkinsonian patients P2 latencies were longer in all conditions while the amplitude of $\mathbf{P} 2$ was about half that of the controls. Latencies on left eye and right eye stimulation are shown in fig 1; this graph is almost identical to that of Bodis Wollner and Yahr. ${ }^{5}$ There was a lack of correlation with age in the patients and in the controls. The sex of the patients or of controls did not play a role in their VEP characteristics nor did type of treatment, duration of disease or duration of treatment in the patients. The interocular difference in the patients was $6.4 \mathrm{~ms}(\mathrm{p}=<0.001)$ and $1.8 \mathrm{~ms}$ in the controls (table 3 ). There was no asymmetry of amplitude despite the interocular difference and indeed further analysis revealed that amplitudes did not correlate with latency. 


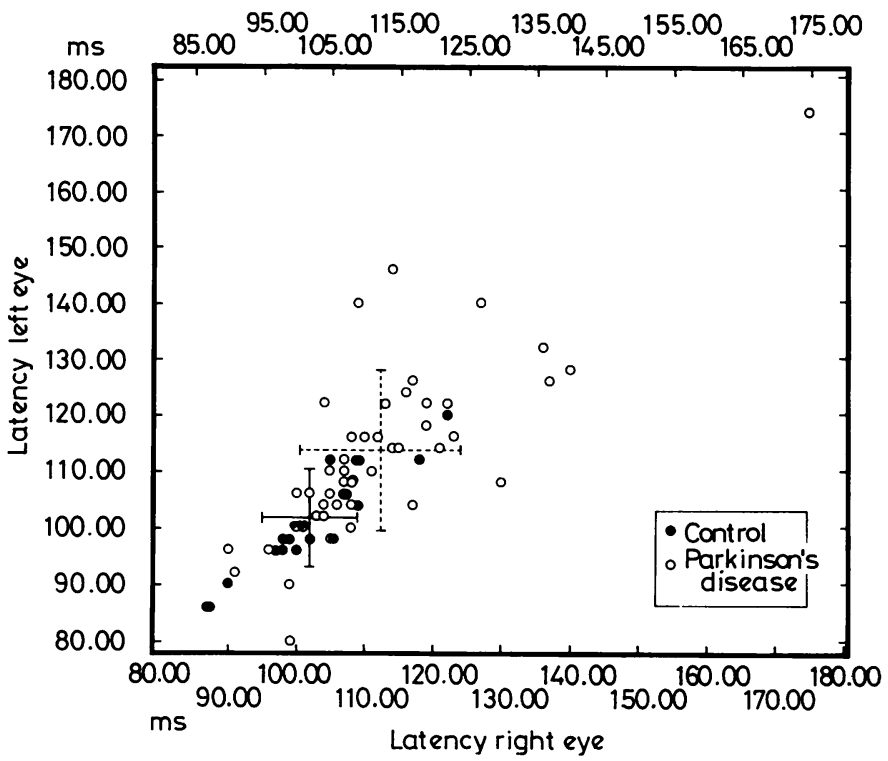

Fig 1 Graph of latency for right eye stimulation plotted against latency for left eye stimulation in patients (open circles) and controls (closed circles) with the standard deviations for each measure.

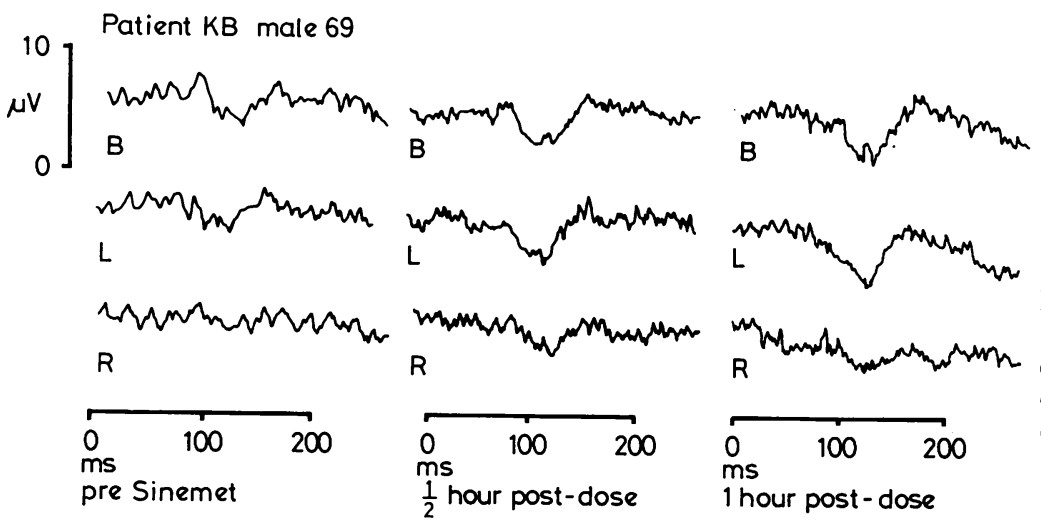

Fig 2 Patient $K B$ (age $69 y r$ ); $V E P$ from binocular, left eye and right eye stimulation demonstrating changes after Sinemet.

Two cases demonstrating effects of treatment on the VEP latency

Case 1 was a 69-year-old man who had had Parkinson's disease for four years. The patient had very severe disability and showed changes in his condition related to the times of dosage. The sequence of evoked potentials obtained (fig 2) demonstrate the effect of a single tablet of Sinemet 110. Half an hour after ingestion the evoked potential became much more clearly defined and of greater amplitude, an effect that was even more marked (except for right eye stimulation) at one hour. Two hours after the dose, the VEP had returned to a state similar to the pre-dose level. Subsequent dosage caused the same sequence of

changes. His condition was improved one hour after each dose, but his attention, awareness and cooperation were not altered. The latency changes were most marked on binocular stimulation.

Case 2 aged 79 years was well controlled on Sinemet 110 for three years and had normal VEP latencies and amplitudes in the first test (fig 3). Six months later he had, for social reasons, stopped taking his tablets and his condition had deteriorated. On retesting the latencies of $P 2$ on right eye and binocular stimulation had increased, while the potential on stimulating his left eye had deteriorated to the extent of becoming unmeasurable, despite normal acuity and ophthalmological examination. 

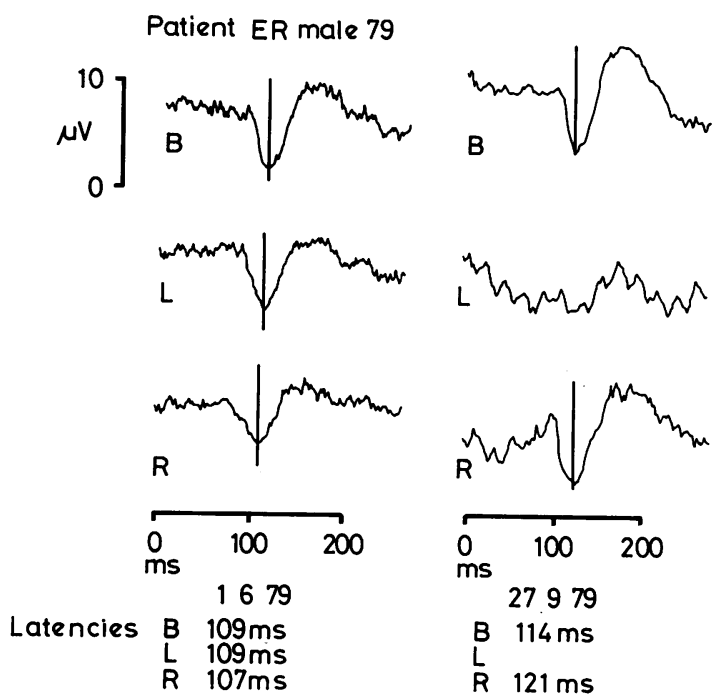

Fig $3 V E P s$ from patient ER. The first recording was taken when he was well controlled on Sinemet. Six months later he stopped his medication and deteriorated clinically. Note the very abnormal left eye waveform and the good but delayed waveform for the right eye.

\section{(2) AUDITORY EVOKED POTENTIALS}

Like the VEP the waveforms obtained on auditory stimulation were odd in form, being less clearly defined than those in the controls, though measurable. The results are summarised on table 4 . There was a delay in the latency of the fifth negative wave NV though not of its amplitude. There was no delay of NI indicating that delay is in the brain stem and not in the end organ. In the thirteen patients who had both AERs and VEPs performed (table 5), three had both delayed VEPs and AERs. Two of these (patients 1 and 2) had had Parkinson's disease for more than 10 years and were on levodopa treatment.

Table 4 AER in Parkinson's disease ( $N V$ wave)

\begin{tabular}{cll}
\hline & Patients $(21)$ & Controls $(12)$ \\
\hline Latency & Rt $5.91 \pm 0.19 \mathrm{~ms}$ & $5.69 \pm 0.18 \mathrm{~ms} \mathrm{p}=0.005$ \\
\pm SD & Lt $5.91 \pm 0.27 \mathrm{~ms}$ & $5.73 \pm 0.27 \mathrm{~ms} \mathrm{p}=0.05$ \\
Amplitude & Rt $0.447 \pm 0.14 \mu \mathrm{v}$ & $0.522 \pm 0.19 \mu \mathrm{v} \mathrm{NS}$ \\
\pm SD & Lt $0.439 \pm 0.18 \mu \mathrm{v}$ & $0.535 \pm 0.23 \mu \mathrm{v} \mathrm{NS}$ \\
& $\mathrm{NI}$ wave \\
Latency & Rt $1.74 \pm 0.12 \mathrm{~ms}$ & $1.74 \pm 0.07 \mathrm{~ms}$ \\
\pm SD & Lt $1.73 \pm 0.16 \mathrm{~ms}$ & $1.74 \pm 0.17 \mathrm{~ms}$ \\
Amplitude & Rt $4.19 \pm 0.25 \mu \mathrm{v}$ & $3.9 \pm 0.12 \mu \mathrm{v}$ \\
\pm SD & Lt $4.185 \pm 0.24 \mu \mathrm{v}$ & $3.98 \pm 0.27 \mu \mathrm{v}$ \\
\hline
\end{tabular}

Table 5 Visual and auditory potentials in Parkinson's disease in individual patients

\begin{tabular}{rllll}
\hline & \multicolumn{2}{l}{$\begin{array}{l}\text { VEP P2 Latency } \\
(\mathrm{ms})\end{array}$} & & \multicolumn{2}{l}{$\begin{array}{l}\text { AER } \\
(\mathrm{ms})\end{array}$} & NV Latency \\
\hline & $R t$ & $L t$ & $R t$ & $L t$ \\
1 & 116 & 124 & $6 \cdot 0$ & $6 \cdot 1$ \\
2 & 129 & 140 & $6 \cdot 0$ & $6 \cdot 0$ \\
3 & 122 & 113 & $6 \cdot 0$ & $6 \cdot 1$ \\
4 & 104 & 104 & $5 \cdot 8$ & $5 \cdot 8$ \\
5 & 116 & 116 & $5 \cdot 8$ & $5 \cdot 8$ \\
6 & 113 & 107 & $5 \cdot 8$ & $5 \cdot 7$ \\
7 & 106 & 102 & $5 \cdot 6$ & $5 \cdot 5$ \\
8 & 100 & 100 & $5 \cdot 8$ & $5 \cdot 7$ \\
9 & 101 & 108 & $5 \cdot 6$ & $5 \cdot 7$ \\
10 & 110 & 116 & $5 \cdot 9$ & $5 \cdot 9$ \\
11 & 103 & 104 & $5 \cdot 8$ & - \\
12 & 108 & 108 & $5 \cdot 9$ & $5 \cdot 6$ \\
13 & 106 & 100 & $5 \cdot 9$ & $5 \cdot 8$ \\
\hline
\end{tabular}

\section{Discussion}

In this study we have confirmed the findings by Bodis Wollner and $\mathrm{Yahr}^{5}$ of a delay in the latency of P2 in the VEP of Parkinsonian patients and the presence of a marked interocular difference. In Bodis Wollner and Yahr's more recent paper, ${ }^{12}$ they categorised their patients according to severity of disease and showed that there was a correlation of prolonged latency of the major-positive wave with clinical severity of disease. In our original presentation $^{11}$ we were unable to do this although some of our patients with very severe and chronic disease did have long latencies. However, the patients with the longest latency $(170 \mathrm{~ms})$ aged 63 years who had had the disease for two years, was on Sinemet and was not severely affected. On the other hand, one patient with severe disease had an extremely short P2 latency. Further follow up, however, has yielded information which suggests that despite treatment the latency does get longer with increased duration of disease though not necessarily with increased severity. For instance, one patient, a female aged 75 years, was first tested on 15 March, 1979. Then her latency for binocular stimulation was $107 \mathrm{~ms}$, left eye $113 \mathrm{~ms}$, right eye $107 \mathrm{~ms}$. When retested on 1 August, 1980 the results were binocular $116 \mathrm{~ms}$, left eye $140 \mathrm{~ms}$ right eye $134 \mathrm{~ms}$. The amplitudes of response were unchanged. She had taken her medication two hours prior to both studies and had not deteriorated clinically. Thus it may be that deterioration in the VEP latency may not proceed at the same rate as clinical deterioration. Degeneration of dopaminergic and other tracts may proceed at different rates throughout the brain, and be reflected by different aspects of dopaminergic functions being affected at different times. Thus, while on the whole a very abnormal VEP, or 
indeed AER, may correlate with severe disease, this may vary widely from individual to individual.

We also found a delay in latency on binocular stimulation and a diminished amplitude of response, in all stimulus conditions, which was symmetrical despite the large interocular latency difference. Mutivariate analysis confirmed that the Parkinsonian patients differed from controls $(\mathrm{p}<0.0001)$ on measures of amplitude and latency of $\mathbf{P 2}$. The strongest discriminator was the amplitude of the major positive wave (A1).

A considerable change occurred when levodopa was administered to the first patient we describe. We have subsequently confirmed this in two other patients. If this can be shown to correlate with clinical status, or even levels of dopamine centrally or levodopa, then this may be a rapid non-invasive way of assessing the amount of dopamine actually arriving at the site of action for this particular function, and how long it stays there. This may be of therapeutic significance in adjusting treatment schedules to provide maximal benefit. The uniocular deterioration in the second patient coupled with the delay on binocular and right eye stimulation is more difficult to explain. Bodis Wollner and Yahr ${ }^{5}$ describe two patients in whom an interocular difference only becomes apparent on treatment. It may be that one eye was more affected by the disease than the other and withdrawal of levodopa had a particularly dramatic effect.

The site of the lesion is of great interest. It is suggested that dopaminergic cells present in the interplexiform layer of the retina may be affected by the disease process and respond to levodopa replacement therapy. Further evidence for the existence of these cells in vertebrates is provided by Watling $e t a^{6}$ and they appear to be pure DI receptors. Other authors ${ }^{13}$ have described a low amplitude in the electro-oculogram which reverted to normal in Parkinson sufferers. ${ }^{13}$ This observation adds support to the possibility that many of the effects we have observed in our patients were retinal. The involvement of such cells would explain the interocular difference but it would be difficult to explain the lack of assymetry in the reduced VEP amplitude on this basis. Other transmitters are involved in Parkinsonism and these may well be involved in some of the amplitude effects. The large VEP amplitude (to flash stimulation) in patients with Down's syndrome can be brought back to normal by administering the 5HT precursor, 5HTP, to the patients. ${ }^{14}$ L tryptophan given to patients with migraine attacks will reduce the large amplitudes of a VEP to flash, but only in those patients whose migraine is ameliorated by the treatment. ${ }^{7} 5 \mathrm{HT}$ is an inhibitory transmitter in the optic nerve and possibly in other areas. Current studies indicate that there is a deficit of 5HT in the patients with Parkinson's disease, ${ }^{15}$ as well as of noradrenaline, $\gamma$ aminobutyric acid, and at least in the putamen acetylcholine receptors. This makes studies as to relative contributions of each of these factors difficult.

It would be interesting to attempt DI receptor inhibition with flupenthixol, chlorpromazine and haloperidol in Parkinsonian patients. There is, in fact, evidence that chlorpromazine does cause a delay in the VEP of normal volunteers. ${ }^{16}$

The delay in the auditory evoked potential suggests a more general involvement by the disease process based on a neurotransmitter deficit or some other factor. The normal latency in the first negative wave which is thought to be derived from the acoustic nerve makes a peripheral lesion unlikely. The fifth negative wave is thought to originate in the inferior colliculus. ${ }^{17}$ So far we have not studied the effects of dosage on the AER in our patients but are in the process of doing so. With current lack of information concerning connections between the inferior colliculus and the visual system it is unjustifiable to speculate on a direct influence. Thus it would seem reasonable to suggest that this effect is due to a generalised neurotransmitter abnormality.

Our studies suggest that the VEP P2 latencies and amplitudes are abnormal in patients with Parkinson's disease and that there is a marked interocular difference in the latencies. The finding that there is also a delayed AER NV wave latency suggests that the lesion may be generalised. Rapid alterations in VEP latency and amplitude following treatment suggest that at least part of the effect observed may be due to a neurotransmitter abnormality although there is not enough information to define this involvement. The use of evoked potential measurement in Parkinson's disorder may be of therapeutic significance.

\section{References}

1 Arden GB, Bodis Wollner I, Halliday AM et al. Methodology of patterned visual stimulation. In: Desmedt JE ed. Visual evoked potentials in man: new developments. Oxford: Clarendon Press, 1977:3-15.

2 Halliday AM, McDonald WI, Muskin J. The visual evoked response in the diagnosis of multiple sclerosis. $\mathrm{Br} \mathrm{Med} J$ 1973; 4:661-4. 
3 Celesia GA, Daly RF. Effects of ageing on visual evoked response. Arch Neurol 1977; 34:403-7.

4 Schafer EWP. McKean CM. Evidence that monoamines influence human evoked potentials. Brain Res 1975; 99:49-58.

5 Bodis Wollner I, Yahr MD. (a) Latency of the pattern evoked potentials in Parkinson's Disease. (11th World Congr Neurology Excerpta Med $1977 ; 427: 126$ ) (b) Measurement of visual evoked potentials in Parkinson's Disease. Brain 1978; 101:661-71.

6 Watling KJ, Dowling JE, Iversen LL. Dopamine receptors in the retina may all be linked to adenylate cyclase. Nature 1979; 281:578-80.

7 Kangasniemi P, Falck B, Langvik Vivi-Ann, Hyppa MT. Levotryptophan treatment in migraine. Headache 1978; 18:161-6.

8 Lehtonen J, Hyppa MT, Kaikola HL, Kangasmiemi P, Lang AH. Visual evoked responses in menstrual migraine. Headache 1979; 19:63-70.

9 Sabelli HC, Giardinan WJ, Barbiol F. Photic evoked cortical potentials; interactions of reserpine, monoamine oxidase inhibitors and dopa. Biol Psychiatry 1971; 3:273-80.

10 Kennard CK, Gawel MJ, Clifford Rose FC, Rudolf $\mathbf{N}$ de $\mathbf{M}$. Visual evoked potentials in migraine patients. In: Friedman AP, Graniger ML, Critchley $\mathrm{M}$ eds. Research and Clinical Studies in Headache. Karger, Basel: 1978:73-80.
11 Gawel MJ, Das PK, Vincent S. Visual and auditory potentials in Parkinson's disease. In: Clifford Rose F, Capildeo R eds. Progress in Parkinson's Disease. New York: Pitman Medical, 1981; 13848.

12 Bodis Wollner I, Yahr M. Visual evoked potentials in Parkinson's disease. In: Clifford Rose F, Capildeo R eds. Progress in Parkinson's disease. New York: Pitman Medical, 1981; 126-37.

13 Economou SG, Stefanis CN. Changes of Electrooculogram. (EOG) in Parkinson's disease. Acta Neurol Scand 1978; 58:44-52.

14 Bigum HB, Dustman RE, Back EC. Visual and sensory evoked responses from mongoloid and normal children. Electroenceph Clin Neurophysiol $1970 ; 28: 576-85$.

15 Reisine JD, Fields JZ, Yamamura HI. Neurotransmitter receptor alterations in Parkinson's disease. Life Sci 1977; 21:335-44.

16 Shagass C. Pharmacology of evoked potentials in man in Psychopharmacology: a review of progress 1957-1967 (ed) DIX Efron. Public Health Service Publication No 1836, US Govt Printing Office, Washington DC 1968; 483-92.

17 Stockard JJ, Rossiter VS. Clinical and pathologic correlates of brain stem auditory response abnormalities. Neurol (Minneap) 1977; 27:316-25. 